Each year in England, over 500,000 carers face the death of the person they were caring for. Preparing for the death of someone close and dealing with its aftermath is one of the most challenging experiences of life, but as with many challenges, it has the capacity to be profound, fulfilling and enriching. For those who were caring for the person, the 'legacies of caring' (Larkin \& Milne, 2017) bring extra dimensions to bereavement. Whether the carer felt prepared for the death or not, the physical, mental, financial and social strain of caring can have lasting impacts well beyond the death.

This presentation will share good practice and ideas to improve outcomes for bereaved carers. Some of these are things that can be put in place before the death, to improve outcomes in bereavement. Others are ways of improving support after the death to make it more sensitive to the legacies of caring'.

The presentation will set out what we know about carers facing and managing bereavement, and some of the recommendations that have been made to improve support. It will summarise the policy context for supporting former carers as a framework.

Drawing on findings from consultations with former carers and those supporting them, it will lay out a series of six aspirations for local areas and services. If these were in place, we would all be able to say 'we get the right support at the right time, before and after the person we are caring for dies'.

Twelve examples of local good practice in supporting carers facing and following bereavement will be shared.

\section{P-246 EVALUATING THE BENEFITS OF MAKE, DO AND MEND}

Lynsey Lawson. St Mary's Hospice, Ulverston, Cumbria

10.1136/bmjspcare-2019-HUKNC.268

Background In December 2017 St Mary's Hospice made a successful application to the Masonic Charitable Foundation Hospice Bereavement Care programme. This application shared our hope to develop a 'Make, Do and Mend workshop' following an internal audit of referrals into the service. This audit identified that the number of bereaved women who came forward for care and support from us, far outnumbered men. We wanted to reach out to these 'missing men'.

Aims Our project aimed to set up a 'Make, Do and Mend' workshop within our retail warehouse, reaching out to bereaved men and women, those caring for their partner or living with their own life-shortening illness. Workshop participants will work alongside each other on a variety of projects and the essence of the group is not building, but the connections, relationships and support between members.

Method The workshop will be led by an experienced retail manager and bereavement staff and volunteers, and will take place in a fully equipped DIY workshop environment. The workshops will run throughout the year on Wednesday and Saturday mornings and participants will be invited to attend eight sessions. Through the use of the Warwick-Edinburgh Wellbeing Scale (WEMWBS) we will seek to evidence an improvement in participants' wellbeing at the end of the eight week programme.

Results Ten workshops have been completed attended by 50 clients (34 men and 16 women). Through the use of the WEMWBS we have identified significant improvements in all 14 areas of the scale as well as clients narratively reporting improvements such as an 80 year old gentleman stating 'The company and overall community feel of the workshop have helped me greatly. We are all in the same boat and support each another through loss'.

\section{P-247 INNOVATION IN BEREAVEMENT CARE - EVALUATING THE IMPACT OF THE HOSPICE ONLINE SUPPORT GROUP}

Lynsey Lawson. St Mary's Hospice, Cumbria, UK

\subsection{6/bmjspcare-2019-HUKNC.269}

Background One of the many things that have changed in the digital age is the way we grieve. While the bereaved have traditionally visited graves or burial sites to talk to deceased loved ones, some are now turning to digital spaces to continue their bonds with the dead. Grieving is once again becoming a community practice with online groups offering support and validation for mourners. As a hospice we recognise the emerging need for online support and as a result we have created our online support group.

Aims Our online support group aims to create:

- A chance for people to share their grief with other bereaved members without feeling unwelcome or uncomfortable;

- A safe place to post poetry, photographs, journals or articles about their loved one who has died;

- A chance for people to feel less lonely in their grief;

- A place to learn coping skills and the importance of self-care.

Method A secure online support group was created in August 2017. Details of the group were shared at initial assessments as well as on the hospice website and on social media in a hope to reach out to the harder to reach groups. The group is supported by trained bereavement volunteers at set times throughout the week.

Results The online support group has been established for 22 months and currently has 82 members ageing from early twenties to late eighties. A further development is that the group now meet monthly at the hospice for a coffee evening. Satisfaction surveys have been completed with the group with one member reporting, 'The group just works for me. We are all in the same boat and support one another, especially as weekends which I find extremely difficult at times'.

\section{P-248 GRIEF IS AS INDIVIDUAL AS A FINGERPRINT. ONE SIZE DOESN'T FIT ALL!}

Lynsey Lawson. St Mary's Hospice, Cumbria, UK

\subsection{6/bmispcare-2019-HUKNC.270}

Background At St Mary's Hospice we recognise grief is unique and individual to the person, but also to each loss. Grieving experiences are unique and can be experienced in different ways. As a hospice we previously adopted the traditional way of formally supporting people who have been bereaved through telephone or face-to-face appointments, however, many people who are bereaved and consider support, choose not to access these traditional services due to their own individual way of grieving and limited alternative support services. As a hospice we recognised it was time to change and develop in line with emerging needs recognising that one size doesn't fit all. 
Aim Our aim was to develop a range of bereavement support options for people to access in a hope to reach out to more people. Our aim was to increase the number of people receiving support from our service by $30 \%$ over a two-year period.

Method Over a two-year period we have developed a broader range of bereavement support services that has scope to encompass individual ways of grieving. These include the traditional one-to-one and telephone support as well as innovative support systems such as a bereavement online support group, monthly bereavement café, Make, Do and Mend, bereavement craft groups as well as offering advice and support sessions at the local library on a weekly basis.

Results We have now established a broader range of bereavement support services and are delighted to be supporting substantially more people. In May 2017 our service caseload for people accessing the service was 96 and by May 2019 this rose to 250 which confirms a $160 \%$ increase in support being provided.

\section{P-249 ENTERING A PERSON'S WORLD RATHER THAN EXPECTING THEM TO ENTER OURS WHEN ADDRESSING PALLIATIVE CARE}

Liz Mathews. Ashgate Hospicecare, Chesterfield, UK

\subsection{6/bmjspcare-2019-HUKNC.271}

Background National and local research studies highlight the negative impact of social isolation, especially in remote areas of high economic and social deprivation (Marmot, 2010). Vulnerable groups e.g. the terminally ill, carers and the bereaved cannot always access mainstream support networks (Steptoe et al., 2013) and can benefit more from accessible, culturally appropriate and varied support supplied within resourceful bereavement hubs in their local community (Social isolation: annual report of the Director Of Public Health For Wirral, 2012-2013).

Aims To develop multi-disciplinary bereavement hubs within isolated communities that provide tailor-made palliative care for pre/post bereavement. This support will embrace cultural diversity, encouraging self-sustaining provision, empowerment and resilience for terminally ill patients and their carers, friends and family, particularly focussing on the more vulnerable in society e.g. young carers. The project aims to lessen social isolation, increase social capital, and address the public health Five Ways To Wellbeing module.

Methods Review literature. Research local/national need. Feedback analysis. Internal consultation. Consultation with relevant local organisations. Funding applications. Secure premises. Recruit and train specialist hub volunteers to provide practical, spiritual and social support. Liaise with other agencies to collaborate. Assessment and personal goal planning. Continuous service review and evaluation, SystmOne reports of service, Core outcomes and individual evaluation questionnaires.

Results Increased coping skills, wellbeing and mental health measured by Core 34 psychological assessment. Greater social activity and increased peer support verified by group activity registers and regular evaluation. Increased referrals and access to service for isolated young carers. Improved multi-agency working. 36 volunteers trained to offer model of support. Increased education regarding self-care achieved by personal goal planning.
Conclusion Evaluation of the pilot hub showed substantial increase in social activity, peer support, resilience and access to local provision aided by increased multi-agency working. It is beneficial for service users and cost-effective for hospice provision. Also, we saw additional coping strategies and autonomy within families, resulting in an improvement in wellbeing.

\section{P-250 DEVELOPING A SUCCESSFUL BEREAVEMENT DROP-IN GROUP}

Tracey Addy, Helen Brown. Kirkwood Hospice, Huddersfield, UK

10.1136/bmjspcare-2019-HUKNC.272

Kirkwood's Bereavement Drop-in has evolved in recent years to its current successful model. Held every two weeks, on average 45 people attend. The Drop-in has been effective, in part due to its informal and relaxed feel. It is arranged in a 'café'-like style which gives us the ability to split people into smaller groups to help people feel more at ease and less intimidated. Although the Drop-in may feel informal, each session is structured and is set around 'themes'. An example might be 'adjustment and change', 'Making sense of my grief' etc.

Themes are discussed with a volunteer support worker or core staff member available to 'steer' the conversation. Peer support is very evident where members feel able to share personal stories - which are obviously encouraged. Halfway through the session, the group is split in order to offer a larger 'structured therapy group'. This group is facilitated by a core team counsellor and a volunteer counsellor.

In an attempt to manage numbers and to encourage people who are newly bereaved we set up a 'new to service' group. This is specifically for people who are newly bereaved or have had a more recent bereavement. It was evident that these people are clearly at a different 'stage' of grieving and they felt intimidated by the large group - who were clearly 'further on' in their grieving process. We offer five sessions in this group as a 'holding' and 'normalising' support.

Feedback for the drop-in session has been positive, and has been an excellent way of engaging the local bereaved community. There is future scope to expand the drop-in event to address isolation and loneliness. Currently, the possibility of an outreach service is being scoped, in order to reach further areas in Kirklees which could possibly involve other members of the hospice multidisciplinary team such as social workers.

\section{Workplace Business and Culture}

\section{P-251 SOCIAL VALUE - WHY IS MEASURING IMPACT IMPORTANT TO HOSPICES?}

Declan Carroll. Peace Hospice Care, Watford, UK

\subsection{6/bmjspcare-2019-HUKNC.273}

Background Commissioners of public services should already take account of evidence impact where appropriate as part of their evaluation when commissioning services. We believe that we should evidence our impact and value for money and be in a stronger position to deliver more to our community i.e. 